\title{
Plano de Controle da Arquitetura NovaGenesis para um Ponto de Interconexão de Tráfego Multi-Arquitetura
}

\author{
Thiago Bueno da Silva ${ }^{1}$, José A. T. Gavazza ${ }^{2}$, Fábio L. Verdi ${ }^{2}$, \\ José A. Suruagy ${ }^{3}$, Juliano Coelho ${ }^{4}$, Flávio Silva ${ }^{4}$, Antônio M. Alberti ${ }^{1}$ \\ ${ }^{1}$ ICT Lab - Instituto Nacional de Telecomunicações (INATEL) \\ Santa Rita do Sapucaí - MG - Brasil \\ ${ }^{2}$ Universidade Federal de São Carlos (UFSCar) \\ Sorocaba - SP - Brasil \\ ${ }^{3}$ Universidade Federal de Pernambuco (UFPE) \\ Recife - PE - Brasil \\ ${ }^{4}$ Universidade Federal de Uberlândia (UFU) \\ Uberlândia - MG - Brasil \\ thiagobueno@gea.inatel.br, gavazza@gmail.com, \{julianoco,flavio\}@ufu.br, \\ verdi@ufscar.br, suruagy@cin.ufpe.br, alberti@inatel.br
}

\begin{abstract}
This article introduces the NovaGenesis architecture to the context of Software Defined Networks with P4. Through the Future Internet Exchange Point project, this architecture can dynamically configure a P4 router that connects two hosts NG. The obtained results prove the feasibility of the prototype once the inserted delays do not impact the NG application notably.
\end{abstract}

Resumo. Este artigo introduz a arquitetura NovaGenesis ao contexto de Software Defined Networks com P4. Por meio do projeto Future Internet Exchange Point, a arquitetura é capaz de configurar dinamicamente um roteador P4 que conecta dois hosts NG. Os resultados obtidos comprovam a viabilidade do protótipo, visto que os atrasos inseridos não impactam muito na aplicação NG.

\section{Introdução}

Com a evolução tecnológica, novas propostas visam otimizar a eficiência da infrastrutura atual de redes de comunicação. Pode-se citar propostas que focam desde a programabilidade de redes pela Software Defined Networks (SDN), bem como propostas mais revolucionárias que almejam redesenhar a própria Internet - pesquisa em Internet do Futuro (IF). Seja qual for o esforço nesta área, a relevância da Internet no mundo atual e futuro é inegável [Qiu et al. 2018]. A sociedade depende desta infraestrutura não mais apenas para diminuir distâncias, mas também como uma matriz econômica capaz de fomentar o desenvolvimento de inúmeros setores, como o financeiro, agrícola e industrial [Silva et al. 2020, Cirillo et al. 2020].

Apesar de seus mais de 50 anos, a Internet sempre apresentou limitações importantes, tais como nomeação perene de entidades, identificação e autenticação de serviços 
e suporte ao roteamento baseado em nomes de conteúdos. Propostas de melhoria evolucionárias não conseguem modificar essa realidade [Pan et al. 2011]. Por outro lado, a completa substituição deste ecossistema por uma outra arquitetura parece inviável por inúmeras questões. Assim, um caminho a se explorar se dá por meio de propostas de cooperação de múltiplas arquiteturas, onde cada arquitetura específica pode oferecer o seu melhor serviço em uma fatia (slice) da rede [Mei et al. 2020]. Propostas como as redes móveis de quinta geração $(5 \mathrm{G})$ e pós-5G fomentam tal visão.

Este artigo introduz a arquitetura NovaGenesis (NG) ao contex to do projeto Future Internet eXchange Point (FIXP). Ao conciliar um controlador NG e um dispositivo roteador baseado na linguagem Programming Protocol-Independent Packet Processor (P4), obtém-se a configuração dinâmica do Plano de Dados. Para tanto, este artigo está estruturado como se segue. A Seção 2 introduz a arquitetura NG. Já a Seção 3 demonstra conceitos básicos de SDN e da arquitetura P4, de modo que a Seção 4 aborde o projeto FIXP. Após isso, a Seção 5 contempla a proposta para conciliar a NG e o FIXP. Em seguida, a Seção 6 demonstra os testes e analisa os resultados obtidos. Por fim, a Seção 7 aponta as principais conclusões do artigo.

\section{NovaGenesis}

NovaGenesis é uma meta arquitetura que combina o estado da arte para conceber um ecossistema convergente de processamento, troca e armazenamento de dados [Alberti et al. 2018]. Desde 2008, este projeto integra paradigmas que buscam mantê-lo relevante em um ambiente tecnologicamente mutável. Alguns pilares derivam de conceitos de redes centradas em informação e serviços, ciclo de vida e gêmeos digitais. Além disso, garante a mobilidade, segurança e confiabilidade em um ambiente que tende a crescer exponencialmente, tanto em termos de dispositivos quanto de dados.

Um dos pilares da NG é o seu sistema de nomeação [Alberti et al. 2016]. Nesta arquitetura, um nome pode ser um identificador ou um localizador, onde as relações entre nomes de dispositivos, processos e domínios são representadas por ligações lógicas entre nomes. Logo, uma entidade possui uma conexão flexível com o seu domínio, a qual é atualizada ao mudar de escopo. Igualmente, o roteamento NG se dá em função dos identificadores de origem e destino, presentes na linha de roteamento NG [Alberti et al. 2016].

Por fim, toda aplicação NG possui uma estrutura que contempla serviços NG básicos, que são essenciais para a comunicação entre processos e/ou aplicações; e especializados, os quais variam conforme a necessidade da aplicação. Assim, micro serviços autossimilares constituem macro serviços NG customizáveis sob demanda. A Tabela 1 expõe brevemente os serviços básicos e especializados NG mais pertinentes a esse artigo.

\section{Software Defined Network e P4}

Lidando com a imutabilidade e rigidez dos dispositivos de redes, a tecnologia SDN provê alta configurabilidade e flexibilidade. Ao dividir a rede em Planos de Dados e Controle, separa-se o tráfego de dados sem comprometer a gerência da rede. Assim, dispositivos comutadores operam sob a orientação de controladores, os quais gerenciam as regras de encaminhamento, fluxo e qualidade de serviço. Ademais, a SDN propicia novos paradigmas de rede sem necessitar de anos de desenvolvimento de dispositivos "convencionais". 
Tabela 1. Serviços básicos e especializados da NovaGenesis.

\begin{tabular}{|c|c|}
\hline Componente NovaGenesis & Funcionalidade \\
\hline $\begin{array}{l}\text { Proxy/Gateway/Controller Service } \\
\text { (PGCS) }\end{array}$ & $\begin{array}{l}\text { Serviço básico que encapsula mensagens NG em padrões de camada de enlace, tais como } \\
\text { Ethernet, LoRa e Wi-Fi; atua como um proxy para representar outros serviços NG dentro } \\
\text { de um mesmo sistema operacional; e realiza o bootstrapping de um domínio. }\end{array}$ \\
\hline Hash Table Service (HTS) & $\begin{array}{l}\text { Serviço básico que armazena ligações entre nomes (name bindings) e conteúdos associa- } \\
\text { dos, se existentes; e atua como um cache de rede. }\end{array}$ \\
\hline $\begin{array}{l}\text { Generic Indirection Resolution Ser- } \\
\text { vice (GIRS) }\end{array}$ & $\begin{array}{l}\text { Serviço básico que seleciona o HTS apropriado para armazenar name bindings e } \\
\text { conteúdos associados. }\end{array}$ \\
\hline Publish/Subscribe Service (PSS) & $\begin{array}{l}\text { Serviço básico que promove a troca de informações entre serviços usando o modelo de } \\
\text { comunicação publica/assina, no qual serviços publicam name bindings e conteúdos para } \\
\text { outros serviços; resolução de nomes, fornecendo a entrega de conteúdos associados via } \\
\text { HTS. }\end{array}$ \\
\hline $\begin{array}{l}\text { Name Resolution and Networking } \\
\text { Cache Service (NRNCS) }\end{array}$ & Serviço básico que engloba o PSS, o HTS e o GIRS em um só programa. \\
\hline ContentApp & $\begin{array}{l}\text { Serviço especializado para distribuição de conteúdo. Um host pode ser configurado como } \\
\text { Fonte de Conteúdo, o qual busca estabelecer um contrato para armazenar os seus dados, } \\
\text { ou Repositório de Conteúdo que oferta a sua capacidade de armazenamento. }\end{array}$ \\
\hline
\end{tabular}

A arquitetura P4 é um exemplo de tecnologia SDN. Esta surgiu como uma evolução do OpenFlow ao propor maior independência de protocolos e dispositivos [Bosshart et al. 2014]. Ao ser agnóstica ao equipamento, deve-se modelar tanto o protocolo a ser processado, quanto o comportamento do dispositivo P4. No entanto, esta não fornece suporte a controladores, os quais são customizáveis conforme cada aplicação.

\section{Future Internet Exchange Point}

O projeto FIXP propõe o roteamento de múltiplos protocolos de comunicação em um mesmo ponto de troca de dados. Para tal fim, comutadores baseados em P4, ou switches (SWes) FIXPs, e programas específicos garantem a comunicação entre os Planos de Dados e Controle. Deste modo, a infraestrutura FIXP é capaz de interconectar e garantir a comunicação de diferentes provedores de serviço da Internet atual e de IF.

A infraestrutura FIXP conta com um Plano de Dados composto por SWes FIXPs que encaminham e processam dados das arquiteturas ETARch, TCP/IP e, agora, NovaGenesis [Gavazza et al. 2020]. Além deste, há uma Camada de Abstração (CA) que realiza a interconexão entre múltiplos SWes FIXPs e múltiplos controladores. Por fim, o Plano de Controle dispõe de controladores que gerenciam o Plano de Dados de maneira dinâmica. Em outras palavras, cada arquitetura utiliza um controlador específico que coordena a topologia física considerando o fluxo de dados de seu protocolo.

Para melhor explanar a arquitetura FIXP, a Figura 1 ilustra o modelo em camadas do projeto, dispondo os programas que atuam em cada estágio. Por sua vez, a Tabela 2 lista e explica sucintamente cada elemento ilustrado na figura em questão.

\section{Proposta de Controlador NovaGenesis para o FIXP}

Atualmente, o PGCS é o melhor serviço NovaGenesis para controlar o FIXP, visto que gerencia elementos programáveis e encapsula mensagens em outros protocolos. Entrementes, o objetivo deste trabalho é estabelecer pilares para um cenário mais complexo para interoperação de outras tecnologias SDN, como OpenFlow e KeyFlow. Esta visão está alinhada com o paradigma bottom-up e, também, com a autossimilaridade da NG.

Primeiramente, o controlador NG precisa obter informações pertinentes à topologia no Plano de Dados. Para fornecer essa visão, 4 bytes reservados foram adicionados ao 


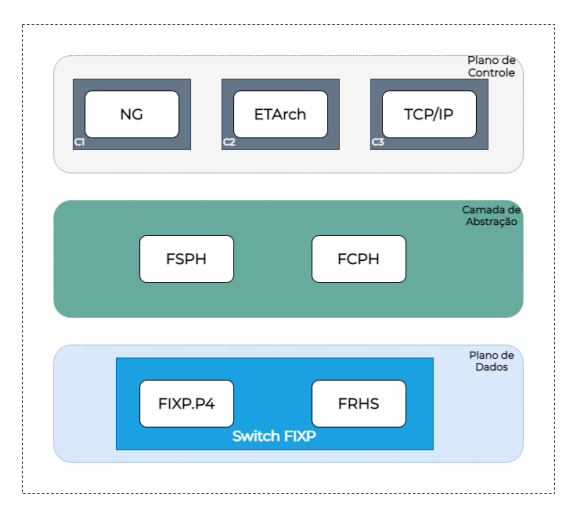

Figura 1. Modelo em Camadas do projeto FIXP.
Tabela 2. Arquitetura FIXP.

\begin{tabular}{|l|l|}
\hline Componente FIXP & Funcionalidade \\
\hline FIXP & Comutador gerido por controladores. \\
\hline FIXP.P4 & Modela os comutadores FIXPs. \\
\hline $\begin{array}{l}\text { FIXP Rule Handler Ser- } \\
\text { vice (FRHS) }\end{array}$ & Traduz as primitivas de controle FIXP. \\
\hline $\begin{array}{l}\text { FIXP Switch Packet } \\
\text { Handler } \text { (FSPH) }\end{array}$ & Interconecta FIXPs a controladores. \\
\hline $\begin{array}{l}\text { FIXP Controller Packet } \\
\text { Handler } \text { (FCPH) }\end{array}$ & Interconecta controladores a FIXPs. \\
\hline Plano de Controle & $\begin{array}{l}\text { Fornece inteligência à infraestrutura com } \\
\text { controladores para cada arquitetura. }\end{array}$ \\
\hline Camada de Abstração & Vincula o Plano de Controle ao de Dados. \\
\hline Plano de Dados & Manipula e roteia dados. \\
\hline
\end{tabular}

cabeçalho de todo pacote NG. Nestes, qualquer FIXP preenche 2 campos para especificar a porta de entrada (PE) que recebeu o pacote NG e o identificador único do comutador, ou Switch Identifier (SWID), ao encaminhar um pacote ao controlador.

No Plano de Controle, o controlador NG mapeia a topologia física ao remontar uma mensagem NG. Por meio de uma tabela interna, o PGCS extrai e registra as informações necessárias à configuração dos FIXPs, como o Host IDdentifier (HID) da origem e, também, o SWID e a PE do FIXP que recebeu um pacote desconhecido. Assim, o HID de destino é comparado com os valores já registrados de HID para determinar se o controlador NG sabe como alcançar o destino solicitado. Caso haja tal conhecimento, uma primitiva de inserção de regra no formato JSON é gerada para configurar o FIXP em questão (esta padronização é uma novidade com a aplicação NG). A Figura 2 ilustra um fluxograma simplificado, considerando apenas ações dos FIXPs e do Controlador NG, omitindo a CA por esta apenas interconectar os Planos de Dados e Controle.

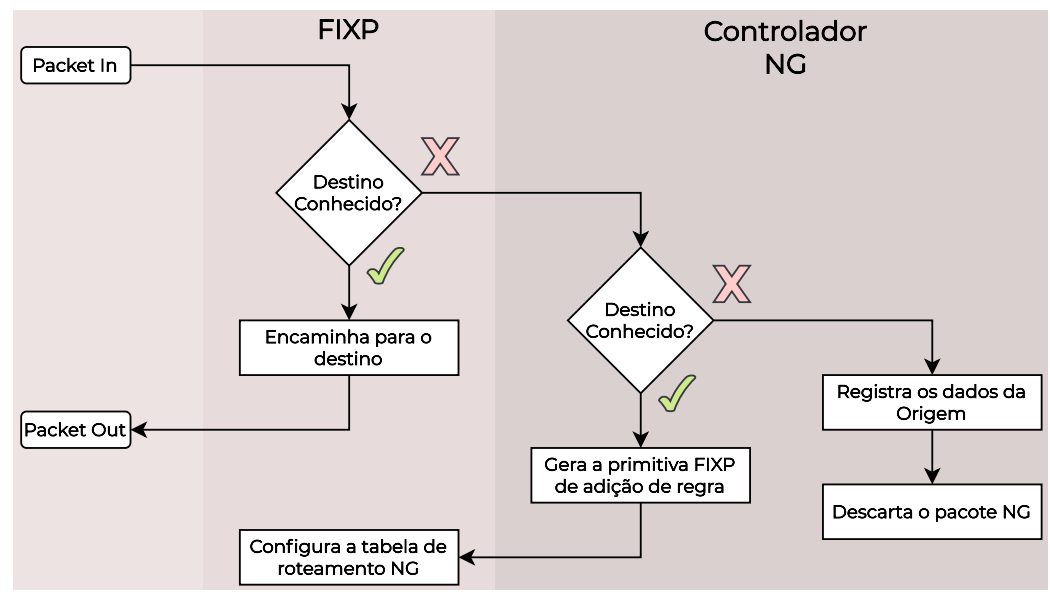

Figura 2. Diagrama de Sequência da operação do FIXP e NovaGenesis.

\section{Análise de Funcionamento}

Para validar a proposta, utiliza-se a aplicação NG de distribuição de conteúdos nomeados. Neste cenário NG, há um repositório de dados que oferece uma capacidade de armazenamento, contando com um PGCS e um ContentApp locais. Além disto, uma fonte de 
dados necessita de um local para armazenar seus dados, dispondo do PGCS, NRNCS e ContentApp. Por meio do VirtualBox, estes dois hosts são conectados por redes virtuais e analisa-se uma topologia com conexão direta entre fonte e repositório; e uma no qual estes dois hosts são conectados pelo FIXP. Logo, a maior topologia utiliza 5 máquinas virtuais (MV) isoladas, onde $2 \mathrm{MVs}$ representam o repositório e a fonte de dados NG e as outras 3 constituem a infraestrutura FIXP, sendo uma para o roteador FIXP, outra para a CA e a última para o Controlador NG com apenas um PGCS. Todas as MVs utilizam o Ubuntu Linux 16.04, com 20 GB de HD, 32 GB de RAM e 2 núcleos de $2.2 \mathrm{GHz}$ do processador Intel Xeon Silver 4114. Essa topologia é apresentada na Figura 3.

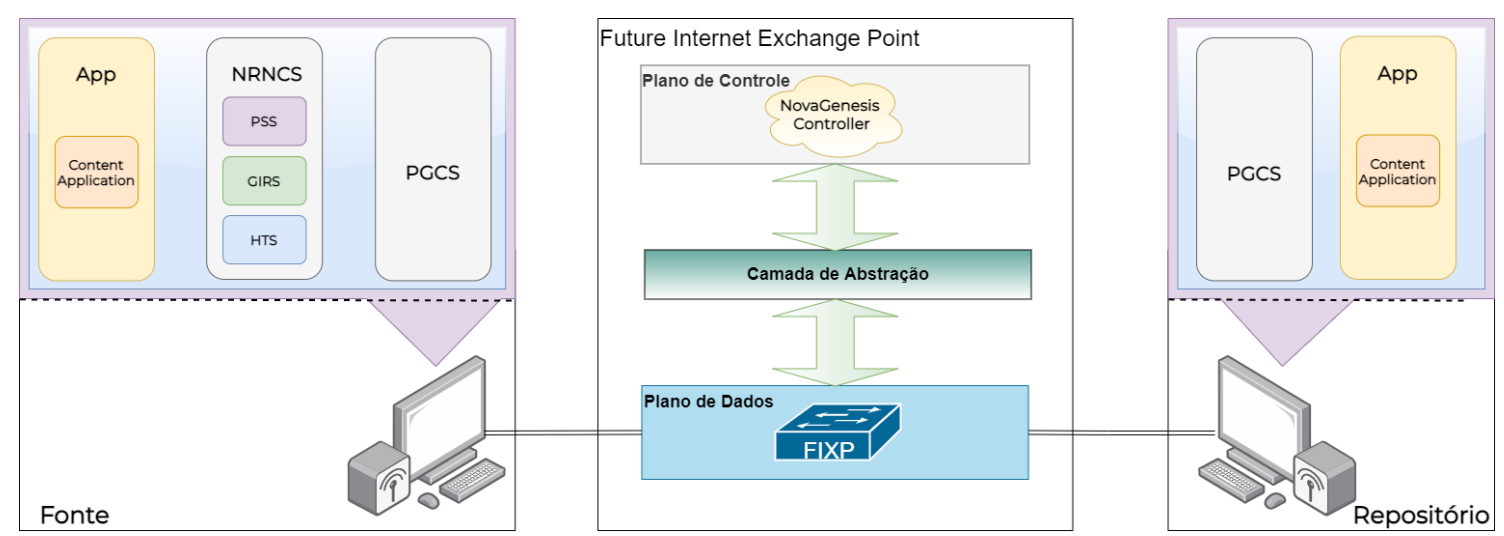

Figura 3. Cenário de Testes FIXP e NG.

Para ambos cenários, 500 fotos de 25, 50 e 100 KB são transferidas. Inicialmente, o tempo de armazenamento (TA), i.e. o intervalo de tempo entre a solicitação de um conteúdo até o instante em que este é totalmente armazenado, é contrastado com e sem FIXP. Junto a isso, investiga-se também o tempo que a rede com FIXP gasta para ser configurada (TC), i.e. o tempo que o FIXP encaminha o primeiro pacote desconhecido ao controlador até o instante em que toda a sua tabela de roteamento está configurada. Por fim, o atraso de processamento inserido pelo FIXP, chamado de overhead, é analisado, sendo empiricamente calculado pela diferença dos valores de TA com e sem FIXP.

A Tabela 3 expõe os resultados de cada cenário. É importante salientar que os dados coletados advêm de uma média aritmética simples de 3 execuções, com intervalo de confiança de $95 \%$. Por meio destes resultados, observa-se que o FIXP impacta minimamente nas aplicações. Por exemplo, o tempo de overhead médio foi de cerca de $69,15 \mathrm{~ms}$. Mesmo que o TC médio seja de 16,39s, este tempo não impacta nas operações NG, visto que um serviço só inicializa ao efetivar um contrato entre os parceiros envolvidos.

Tabela 3. Resultados obtidos.

\begin{tabular}{|l|l|l|l|l|}
\hline Cenário & TA sem FIXP & TA com FIXP & TC com FIXP & Overhead \\
\hline 500 fotos de $25 \mathrm{~KB}$ & $880,9 \mathrm{~ms} \pm 127,69 \mathrm{~ms}$ & $918,76 \mathrm{~ms} \pm 129,03 \mathrm{~ms}$ & $15,259 \mathrm{~s}$ & $37,87 \mathrm{~ms}$ \\
\hline 500 fotos de $50 \mathrm{~KB}$ & $1,032 \mathrm{~s} \pm 133,87 \mathrm{~ms}$ & $1,135 \mathrm{~s} \pm 140,6 \mathrm{~ms}$ & $18,574 \mathrm{~s}$ & $102,82 \mathrm{~ms}$ \\
\hline 500 fotos de $100 \mathrm{~KB}$ & $1,314 \mathrm{~s} \pm 158,57 \mathrm{~ms}$ & $1,381 \mathrm{~s} \pm 157,47 \mathrm{~ms}$ & $15,33 \mathrm{~s}$ & $66,76 \mathrm{~ms}$ \\
\hline
\end{tabular}

\section{Conclusão}

Este artigo introduz a arquitetura NovaGenesis ao projeto FIXP. Esse é o primeiro trabalho que demonstra métricas de desempenho da NG com esta infraestrutura. Os resultados 
apresentados comprovam a proposta e validam o seu desempenho, uma vez que os atrasos inseridos não impactam significativamente no desempenho das aplicações.

Este trabalho estabelece pilares para um ecossistema NG de interoperação SDN, onde a NG pode ser uma arquitetura que gerencie slices e elementos programáveis de rede. Ao explorar o projeto FIXP, a NG configura nativamente elementos P4 programáveis na rede. Trabalhos futuros explorarão mais essa cooperação, contemplando topologias mais complexas e propondo metodologias mais precisas de validação.

\section{Agradecimentos}

Este trabalho foi parcialmente financiado pela RNP, com recursos do MCTI, processo No 01245.010604/2020-14, sob o projeto Sistemas de Comunicações Móveis de $6^{\text {a }}$ Geração (6G) do Centro de Referência em Radiocomunicações (CRR) do Instituto Nacional de Telecomunicações - Inatel, Brasil. Ademais, contou com recursos da Fundação de Amparo à Pesquisa do Estado de São Paulo (FAPESP) pela chamada \#2015/24518-4 destinados ao projeto FIXP. Os autores também agradecem CAPES, o CNPq e a FAPEMIG.

\section{Referências}

Alberti, A., Casaroli, M., Righi, R., and Singh, D. (2018). Introducing novagenesis as a novel distributed system-based convergent information architecture. In NatureInspired Networking Theory and Applications, chapter 4, pages 88-144. CRC Press.

Alberti, A., Casaroli, M., Singh, D., and Righi, R. (2016). Naming and name resolution in the future internet: Introducing the novagenesis approach. Future Generation Computer Systems, 67.

Bosshart, P., Daly, D., Gibb, G., Izzard, M., McKeown, N., Rexford, J., and et al. (2014). P4: Programming Protocol-Independent Packet Processors. SIGCOMM Comput. Commun. Rev., 44(3):87-95.

Cirillo, F., Gómez, D., Diez, L., Elicegui Maestro, I., Gilbert, T. B. J., and Akhavan, R. (2020). Smart city iot services creation through large-scale collaboration. IEEE Internet of Things Journal, 7(6):5267-5275.

Gavazza, J. A. T., Melo, J. C., da Silva, T. B., Alberti, A. M., Rosa, P. F., de Oliveira Silva, F., Verdi, F. L., and Suruagy, J. A. (2020). Future internet exchange point (fixp): Enabling future internet architectures interconnection. In Barolli, L., Amato, F., Moscato, F., Enokido, T., and Takizawa, M., editors, Advanced Information Networking and Applications, pages 703-714, Cham. Springer International Publishing.

Mei, C., Liu, J., Li, J., Zhang, L., and Shao, M. (2020). 5g network slices embedding with sharable virtual network functions. Journal of Comm. and Nets, 22(5):415-427.

Pan, J., Paul, S., and Jain, R. (2011). A survey of the research on future internet architectures. IEEE Communications Magazine, 49(7):26-36.

Qiu, T., Chen, N., Li, K., Atiquzzaman, M., and Zhao, W. (2018). How Can Heterogeneous Internet of Things Build Our Future: A Survey. IEEE Communications Surveys Tutorials, 20(3):2011-2027.

Silva, T. B. d., Morais, E. S. d., Almeida, L. F. F. d., Rosa Righi, R. d., and Alberti, A. M. (2020). Blockchain and Industry 4.0: Overview, Convergence, and Analysis, pages 27-58. Springer Singapore, Singapore. 